\title{
Effects of losartan combined with exercise training in spontaneously hypertensive rats
}

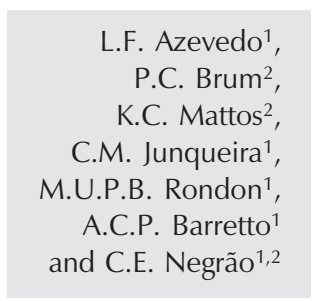

\section{Correspondence}

C.E. Negrão

Instituto do Coração (InCor)

Unidade de Reabilitação

Cardiovascular e Fisiologia

do Exercício

Av. Dr. Enéas C. Aguiar, 44

Bloco II, andar -1

05403-000 São Paulo, SP

Brasil

Fax: +55-11-3069-5043

E-mail: cndnegrao@incor.usp.br

Research supported by Merck \& Co.,

Inc., Rahway, NJ, USA, and supported

in part by Fundação Zerbini,

São Paulo, SP, Brazil. C.E. Negrão

received a research scholarship from

Merck \& Co. to develop the present

study.

Publication supported by FAPESP.

$\ldots \ldots \ldots \ldots \ldots \ldots$

Received June 25, 2002

Accepted July 23, 2003

\author{
${ }^{1}$ Instituto do Coração (InCor), Hospital das Clínicas, Faculdade de Medicina, \\ and ${ }^{2}$ Escola de Educação Física e Esportes, Universidade de São Paulo, \\ São Paulo, SP, Brasil
}

\begin{abstract}
We investigate whether combined treatment with losartan, an angiotensin II receptor blocker, and exercise training (ET) in spontaneously hypertensive rats (SHR) would have an additive effect in reducing hypertension and improving baroreflex sensitivity when compared with losartan alone. Male SHR (8 weeks old) were assigned to 3 groups: sedentary placebo ( $\mathrm{SP}, \mathrm{N}=16$ ), sedentary under losartan treatment ( $\mathrm{SL}, \mathrm{N}=11 ; 10 \mathrm{mg} \mathrm{kg}^{-1}$ day $^{-1}$, by gavage), and ET under losartan treatment (TL, $\mathrm{N}=10$ ). ET was performed on a treadmill 5 days/week for $60 \mathrm{~min}$ at $50 \%$ of peak $\mathrm{VO}_{2}$, for 18 weeks. Blood pressure (BP) was measured with a catheter inserted into the carotid artery, and cardiac output with a microprobe placed around the ascending aorta. The baroreflex control of heart rate was assessed by administering increasing doses of phenylephrine and sodium nitroprusside $(i v)$. Losartan significantly reduced mean $\mathrm{BP}(178 \pm 16$ vs 132 $\pm 12 \mathrm{mmHg})$ and left ventricular hypertrophy $(2.9 \pm 0.4$ vs $2.5 \pm 0.2$ $\mathrm{mg} / \mathrm{g}$ ), and significantly increased baroreflex bradycardia and tachycardia sensitivity $(1.0 \pm 0.3$ vs $1.7 \pm 0.5$ and $2.0 \pm 0.7$ vs $3.2 \pm 1.7 \mathrm{bpm} /$ $\mathrm{mmHg}$, respectively) in SL compared with SP. However, losartan combined with ET had no additional effect on BP, baroreflex sensitivity or left ventricular hypertrophy when compared with losartan alone. In conclusion, losartan attenuates hypertension and improves baroreflex sensitivity in SHR. However, ET has no synergistic effect on BP in established hypertension when combined with losartan, at least at the dosage used in this investigation.
\end{abstract}

\section{Introduction}

Hypertension is a major population health problem and is a precursor of various forms of cardiovascular disease such as congestive heart failure $(1,2)$ but is considered a modifiable risk
Key words

- Spontaneously hypertensive rats

- Exercise training

- Losartan

- Baroreflex sensitivity factor for morbidity and mortality. Since a reduction in resting arterial blood pressure is associated with a decrease in risk factors, several pharmacological and nonpharmacological therapies for hypertension management have been assessed in many studies (3-6). 
Both exercise training (ET) and drug therapy for hypertension can be independently effective, and have been associated with decreased morbidity and mortality in hypertension (5-8). While losartan, one of the most used $\mathrm{AT}_{1}$ receptor blockers, limits the development of hypertension $(9,10)$ and decreases blood pressure levels in spontaneously hypertensive rats (SHR) and in patients with essential hypertension (11-14), low-intensity ET attenuates hypertension but fails to reduce blood pressure to normotensive levels $(15,16)$. In addition, both losartan treatment and ET independently improve the impaired baroreflex sensitivity in SHR (1719) and lead to cardiac remodeling with reduction of left ventricular hypertrophy $(7,12,13,20-23)$.

Even though the separate effects of losartan and ET on hypertension management and therapy have been extensively studied, the benefits achieved by the combination of these two procedures in terms of blood pressure and arterial baroreflex sensitivity are unclear. Some previous reports demonstrated no additional benefit of ET combined with drug therapy such as adrenergic receptor blockers and diuretics (24-26), while others found a synergistic effect of ET when combined with $\alpha$-adrenoceptor blockade and diuretics in SHR (16). Recent studies have demonstrated that losartan combined with ET improves renal function in renal failure rats (27) and reduces insulin resistance in a rat model of type 2 diabetes mellitus (28).

In the present study, we tested the hypothesis that combined treatment of SHR with losartan and ET would have an additive effect in reducing high blood pressure and improving the impaired arterial baroreflex when compared with losartan alone. In addition, we also performed hemodynamic measurements to evaluate the mechanisms underlying the expected combined effect of losartan and ET in reducing arterial pressure of SHR.

\section{Material and Methods}

\section{Animal care}

Male SHR (School of Physical Education and Sports, University of São Paulo), 8 weeks old, $211 \pm 5 \mathrm{~g}$ body weight, 4 rats/ cage, were fed standard laboratory chow and received water ad libitum in a temperaturecontrolled room $\left(22-24^{\circ} \mathrm{C}\right)$ with a $12: 12$-h light/dark cycle. The rats were randomly assigned to 3 groups: sedentary placebo (SP, $\mathrm{N}=16$ ), sedentary under losartan treatment (SL, N = 11), and ET under losartan treatment (TL, $N=10$ ). The study was conducted according to the Ethical Principles in animal research adopted by the Brazilian College of Animal Experimentation.

\section{Exercise training and losartan treatment protocols}

ET was started when the rats were 8 weeks old and consisted of 18 weeks of running on a motor treadmill (ESD model 01 FUNBEC, São Paulo, SP, Brazil), 5 days/ week, for 60 min and gradually progressing towards $50 \%$ of peak oxygen uptake (peak $\mathrm{VO}_{2} ; 18-20 \mathrm{~m} / \mathrm{min}$ ). The sedentary rats were handled every day for habituation to the experimental protocols. Peak $\mathrm{VO}_{2}$ was measured by expired gas analysis during a progressive exercise testing on a motor treadmill with $5 \mathrm{~m} / \mathrm{min}$ increments $(5-40 \mathrm{~m} / \mathrm{min}$ ) every 3 min and no grade until exhaustion. Rats were considered to be exhausted when they were unable to keep running at the treadmill speed. Oxygen and carbon dioxide concentrations were analyzed by the Scholander microtechnique (29). Peak $\mathrm{VO}_{2}$ was determined at the maximum speed reached during the progressive exercise testing. The treadmill exercise test was repeated after 8 weeks of ET in order to adjust training intensity and at the end of the experimental protocol.

Experimental rats were treated with the 
$\mathrm{AT}_{1}$ receptor blocker losartan $\left(10 \mathrm{mg} \mathrm{kg}{ }^{-1}\right.$ day $^{-1}$ ) and control rats received tap water. Both losartan and tap water were administered by gavage. Losartan treatment and ET were continued throughout the 18 weeks of the experimental protocol. Losartan was provided by Merck and Co. (Rahway, NJ, USA).

Cardiac output. Rats were anesthetized with ketamine $(50 \mathrm{mg} / \mathrm{kg}$, ip) and xylazine $(10 \mathrm{mg} / \mathrm{kg}, i p)$ and intubated with a tracheal catheter (catheter placement unit $2.1 \mathrm{~mm}$ in diameter x $5.1 \mathrm{~cm}$ in length) for mechanical ventilation (Havard Rodent Ventilator, model 683, South Natick, MA, USA) at a respiratory rate of 70 breaths/min and tidal volume of $2.5 \mathrm{ml} / \mathrm{breath}$ throughout the surgical procedures. A transverse incision was performed on the right side of the chest wall in the second intercostal space just below the axillary area. The pectoral musculature was carefully opened and right thoracotomy was performed to access the ascending aorta. An ultrasonic perivascular flow probe (2.5 SL; Transonic System Inc., Ithaca, NY, USA) was placed around the ascending aorta just above the coronary arteries and emerged through the back of the rat. Body temperature was maintained stable at $37^{\circ} \mathrm{C}$ by heating. After surgery, rats received a veterinary pentabiotic (Fort Dodge Saúde Animal Ltda., Campinas, SP, Brazil) and were returned to individual cages. Rats were allowed to recover from surgery for 12 days. Losartan treatment and ET were interrupted for 1 and 5 days, respectively, after surgery. During the experimental session, the flow probe was connected to an ultrasonic flowmeter (Transonic, model T206) and connected to a 16channel analog-to-digital converter (Stoelting Co., Wood Dale, IL, USA) and finally to a microcomputer for direct cardiac output measurement.

Blood pressure and heart rate. Tail-cuff blood pressure was recorded before (8-weekold rats) and after (26-week-old rats) ET and losartan treatment using a computerized tailcuff system. Blood pressure measurements obtained on two consecutive days were averaged and the mean was considered as the tail-cuff blood pressure for the animal. Twenty-four hours after the last ET session, two catheters $(0.05 \mathrm{~mm}$ in internal diameter $)$ were implanted, one into the carotid artery and another into the jugular vein, and both emerged through the back of the rat. This surgical procedure was performed under ketamine $(50 \mathrm{mg} / \mathrm{kg}, i p)$ and xylazine $(10 \mathrm{mg} / \mathrm{kg}$, $i p$ ) anesthesia for direct blood pressure measurements and drug injection. The arterial catheter was connected to a strain-gauge transducer (P23 Db; Gould-Statham, Oxnard, CA, USA) and fed into an amplifier (General Purpose Amplifier, Stemtech, Wood Dale, IL, USA). Thus, the signal was connected to a 16-channel analog-to-digital converter (Stoelting) and then to a microcomputer for direct blood pressure measurements. Heart rate measurement was determined on line, derived from the blood pressure recordings. Stroke volume was calculated from cardiac output and heart rate. Total peripheral vascular resistance was calculated from mean blood pressure and cardiac output. Stroke volume, cardiac output, and total peripheral vascular resistance were corrected for $100 \mathrm{~g}$ of body weight.

Arterial baroreflex sensitivity. Arterial baroreflex sensitivity was measured by repeated $i v$ bolus injections $(0.1 \mathrm{ml})$ of phenylephrine ( 0.25 to $4 \mu \mathrm{g} / \mathrm{kg}$; Sigma, St. Louis, MO, USA) and sodium nitroprusside (0.1 to $4 \mu \mathrm{g} / \mathrm{kg}$; Sigma). Heart rate response to mean arterial pressure changes was calculated for each dose. No subsequent injections were made until the recorded parameters had returned to the preinjection level. Two different procedures were performed to determine the baroreflex sensitivity: i) calculation of the mean ratios of heart rate responses to mean arterial pressure changes for each dose used per rat (mean index - $\triangle \mathrm{HR} / \Delta \mathrm{MAP}$ ), and ii) linear regression analysis (30), whereby the coefficient (slope) of each animal's regression line was averaged and taken as an 
index of baroreflex sensitivity.

Left ventricular hypertrophy. Gross examination of the heart was done by determining the left ventricular weight to body weight ratio.

\section{Experimental protocol}

Losartan treatment and ET were started in 8-week-old rats and lasted 18 weeks. After this period, rats were submitted to a maximal exercise test to evaluate the peak $\mathrm{VO}_{2}$. Twenty-four hours later, a flow probe was placed around the aorta of the animals, which were allowed to recover for 12 days. During the recovery period, losartan treatment and ET were interrupted for 1 and 5 days, respectively, with drug treatment and ET being resumed thereafter. Following the 12-day recovery period, arterial and venous catheters were inserted for blood pressure recording and drug injections, respectively. Twenty-four hours after catheterization, cardiac output and blood pressure were monitored simultaneously for $30 \mathrm{~min}$ in quiet, conscious rats on a beat-to-beat basis (AT/ CODAS, Dataq Instruments Inc., Akron, OH, USA). Subsequently, arterial baroreflex sen-

Table 1. Body weight, peak oxygen uptake (peak $\mathrm{VO}_{2}$ ) and tail-cuff blood pressure in sedentary placebo (SP), sedentary under losartan treatment (SL) and exercise training under losartan treatment (TL) spontaneously hypertensive rats, before and after 18 weeks of losartan treatment and exercise training.

\begin{tabular}{lccc}
\hline & SP & SL & $T L$ \\
\hline $\begin{array}{l}\text { Body weight (g) } \\
\text { Before }\end{array}$ & $226.1 \pm 30.7$ & $203.6 \pm 15.7^{*}$ & $194.3 \pm 21.1^{*}$ \\
$\quad$ After & $318.8 \pm 30.6^{* *}$ & $302.2 \pm 26.5^{* *}$ & $304.5 \pm 27.8^{* *}$ \\
Peak VO $2\left(\mathrm{ml} \mathrm{kg}^{-1} \mathrm{~min}^{-1}\right)$ & & & \\
$\quad$ Before & $85.0 \pm 4.3$ & $84.6 \pm 5.2$ & $87.6 \pm 5.9$ \\
$\quad$ After & $62.2 \pm 6.3^{* *}$ & $64.0 \pm 6.4^{* *}$ & $88.9 \pm 8.4^{+}$ \\
Tail-cuff pressure $(\mathrm{mmHg})$ & & \\
$\quad$ Before & $155.0 \pm 22.0$ & $162.0 \pm 17.9$ & $157.3 \pm 22.6$ \\
$\quad$ After & $197.9 \pm 26.2^{* *}$ & $167.6 \pm 18.3$ & $173.6 \pm 20.2$ \\
\hline
\end{tabular}

Data are reported as means $\pm \mathrm{SD}$. ET = exercise training.

${ }^{*} \mathrm{P}<0.05$ vs SP group before losartan treatment and $\mathrm{ET}$; ${ }^{*} \mathrm{P}<0.01$ vs before losartan treatment and $\mathrm{ET} ;+\mathrm{P} \leq 0.05$ vs sedentary groups (SP and $\mathrm{SL}$ ) after losartan treatment and ET (repeated measures ANOVA). sitivity was assessed in all 3 groups. After evaluation the rats were anesthetized with ketamine $(50 \mathrm{mg} / \mathrm{kg}$, ip) and xylazine $(10$ $\mathrm{mg} / \mathrm{kg}$, ip) until complete cardiac arrest, and the heart was excised and weighed for gross examination.

\section{Statistical analysis}

All data are reported as means $\pm \mathrm{SD}$. Comparisons of direct blood pressure, heart rate, cardiac output index, systolic volume index, total peripheral vascular resistance index, arterial baroreflex sensitivity, and left ventricular weight to body weight ratio were performed by one-way ANOVA followed by the post hoc Scheffé test. For body weight, peak $\mathrm{VO}_{2}$ and tail-cuff blood pressure, comparisons were made by repeated measures ANOVA followed by the post hoc Scheffé test. The level of significance was set at $\mathrm{P} \leq$ 0.05

\section{Results}

\section{Body weight, peak oxygen uptake and tail-} cuff blood pressure

The data concerning body weight, peak $\mathrm{VO}_{2}$ and tail-cuff blood pressure before and after treatment with losartan and ET are shown in Table 1. The SP group had higher body weight than the SL and TL groups at the beginning of the study. After 18 weeks of the experimental protocol, body weight increased similarly in all 3 groups studied. At the beginning of the study, peak $\mathrm{VO}_{2}$ was similar in all 3 groups studied before the losartan and ET protocols. However, after 18 weeks of the experimental protocol, peak $\mathrm{VO}_{2}$ decreased in the SP and SL groups, but not in the TL group, in which peak $\mathrm{VO}_{2}$ remained similar to that found before treatment with losartan and ET. In addition, peak $\mathrm{VO}_{2}$ was significantly higher in the TL than in the SP and SL groups after the experimental protocol. At the beginning of the study, 
no differences in tail-cuff blood pressure levels were found among groups. However, after 18 weeks of the experimental protocol, the SP group presented a higher tail-cuff blood pressure than before the experimental protocol.

\section{Hemodynamic responses and left ventricular hypertrophy}

The data concerning resting blood pressure, cardiac output index, heart rate, stroke volume index, total peripheral vascular resistance index, and left ventricular weight to body weight ratio after 18 weeks of the experimental protocol are shown in Table 2. Consistent with tail-cuff blood pressure measurements, losartan treatment significantly decreased systolic, diastolic and mean blood pressure levels. However, there was no additional effect of ET on systolic, diastolic or mean blood pressure, since no additional blood pressure reduction was observed in TL rats compared with SL. Losartan induced no change in cardiac output index, heart rate or stroke volume index. In fact, losartan significantly reduced the total peripheral vascular resistance index and the left ventricular weight to body weight ratio. ET had no additive effect on the reduction of total peripheral vascular resistance index and left ventricular weight to body weight ratio provoked by losartan.

\section{Arterial baroreflex sensitivity}

The data concerning arterial baroreflex sensitivity in terms of bradycardia and tachycardia analyzed by mean index are shown in Table 3 and Figure 1. The linear regression analysis is shown in Table 3 and Figure 2. Losartan significantly increased arterial baroreflex sensitivity determined by mean index for bradycardia in both groups (SL and TL); however, tachycardia was improved only in the SL group. Nevertheless, losartan significantly increased arterial baroreflex sensitiv- ity determined by linear regression analysis for both bradycardia and tachycardia. Moreover, there was no synergistic effect of ET on arterial baroreflex sensitivity for bradycardia and tachycardia.

\section{Discussion}

The main findings of the present study were: i) losartan treatment decreased resting

Table 2. Hemodynamic responses and left ventricular weight to body weight ratio in sedentary placebo (SP), sedentary under losartan treatment (SL) and exercise training under losartan treatment (TL) spontaneously hypertensive rats, after 18 weeks of losartan treatment and exercise training.

\begin{tabular}{lccc}
\hline & $\mathrm{SP}$ & $\mathrm{SL}$ & $\mathrm{TL}$ \\
\hline $\mathrm{SBP}(\mathrm{mmHg})$ & $207.6 \pm 16$ & $\begin{array}{c}151.6 \pm 11^{*} \\
(-27 \%)\end{array}$ & $\begin{array}{c}149.2 \pm 17^{*} \\
(-28 \%)\end{array}$ \\
DBP (mmHg) & $149.6 \pm 18$ & $\begin{array}{c}111.2 \pm 15^{*} \\
(-26 \%)\end{array}$ & $\begin{array}{c}108.4 \pm 12^{*} \\
(-28 \%)\end{array}$ \\
MBP (mmHg) & & $\begin{array}{c}131.7 \pm 12^{*} \\
178.1 \pm 16\end{array}$ & $\begin{array}{c}128.8 \pm 14^{*} \\
\left.\text { COI (ml min-1 } 100 \mathrm{~g} \mathrm{~g}^{-1}\right)\end{array}$ \\
HR (bpm) & $20.5 \pm 1.7$ & $22.4 \pm 2.1$ & $19.8 \pm 3.7$ \\
SVI (ml beat $\left.{ }^{-1} 100 \mathrm{~g} \mathrm{~g}^{-1}\right)$ & $322.6 \pm 28$ & $323.4 \pm 31$ & $308.9 \pm 32$ \\
TPVRI (mmHg ml $\left.\mathrm{min}^{-1} 100 \mathrm{~g}^{-1}\right)$ & $0.065 \pm 0.01$ & $0.070 \pm 0.01$ & $0.065 \pm 0.01$ \\
LVW:BW (mg/g) & $2.9 \pm 0.4$ & $0.669 \pm 0.2^{*}$ & $0.728 \pm 0.1^{*}$ \\
\hline
\end{tabular}

Data are reported as means $\pm \mathrm{SD}$. $\mathrm{COI}=$ cardiac output index, $\mathrm{DBP}=$ diastolic blood pressure, $\mathrm{HR}=$ heart rate, $\mathrm{LVW}: \mathrm{BW}=$ left ventricular weight to body weight ratio, $\mathrm{MBP}=$ mean blood pressure, $\mathrm{SBP}=$ systolic blood pressure, $\mathrm{SVI}=$ stroke volume index, TPVRI $=$ total peripheral vascular resistance index.

${ }^{*} \mathrm{P}<0.05$ vs SP (one-way ANOVA).

Table 3. Arterial baroreflex for bradycardia and tachycardia by mean index (bpm/ $\mathrm{mmHg}$ ) and linear regression analysis coefficient (slope) in sedentary placebo (SP), sedentary under losartan treatment (SL) and exercise training under losartan treatment (TL) spontaneously hypertensive rats, after 18 weeks of losartan treatment and exercise training.

\begin{tabular}{lccc}
\hline & SP & SL & TL \\
\hline $\begin{array}{l}\text { Mean index (bpm/mmHg) } \\
\text { Bradycardia }\end{array}$ & $1.03 \pm 0.5$ & $1.67 \pm 0.7^{*}$ & $2.04 \pm 1^{*}$ \\
Tachycardia & $2.02 \pm 0.8$ & $3.22 \pm 1.3^{*}$ & $2.62 \pm 0.9^{+}$ \\
& & & \\
Linear regression slope (bpm/mmHg) & & & \\
$\quad$ Bradycardia & $-0.71 \pm 0.5$ & $-1.73 \pm 0.4^{*}$ & $-2.31 \pm 1.3^{*}$ \\
Tachycardia & $-1.09 \pm 2.0$ & $-2.74 \pm 0.7^{*}$ & $-2.61 \pm 0.6^{*}$ \\
\hline
\end{tabular}

Data are reported as means $\pm \mathrm{SD}$

${ }^{*} \mathrm{P}<0.05$ vs $\mathrm{SP} ;{ }^{+} \mathrm{P}=0.001$ vs $\mathrm{SL}$ (one-way ANOVA). 
systolic, diastolic and mean blood pressure, and total peripheral vascular resistance index in SHR, ii) losartan improved arterial baroreflex sensitivity for bradycardia and tachycardia in SHR, iii) losartan reduced left ventricular weight to body weight ratio in SHR, and iv) ET associated with losartan caused no additional effect on blood pressure, arterial baroreflex sensitivity for bradycardia and tachycardia or left ventricular weight to body weight ratio in SHR.

The higher peak $\mathrm{VO}_{2}$ in the ET group compared with the sedentary groups showed the effectiveness of the ET protocol. This result supports our previous findings that there is no decrease in peak $\mathrm{VO}_{2}$ in exercisetrained SHR (15). In fact, exercise training attenuated the decrease in peak $\mathrm{VO}_{2}$ corrected to body weight during the rat's life span as previously demonstrated by others (16).

Figure 1. Arterial baroreflex sensitivity determined by mean index ( $\triangle \mathrm{HR} / \Delta \mathrm{MAP})$ for bradycardia and tachycardia in sedentary placebo (SP), sedentary under losartan treatment (SL) and exercise training under losartan treatment (TL) spontaneously hypertensive rats, after 18 weeks of losartan treatment and exercise training. Data are reported as means $\pm \mathrm{SD}$. ${ }^{*} \mathrm{P}<0.001$ vs $\mathrm{SP}$ $+P<0.001$ vs $S L$ lone-way ANOVA).

Figure 2. Arterial baroreflex sensitivity determined by linear regression coefficient (slope) for bradycardia and tachycardia in sedentary placebo (SP), sedentary under losartan treatment $(\mathrm{SL})$ and exercise training under losartan treatment (TL) spontaneously hypertensive rats, after 18 weeks of losartan treatment and exercise training. $\mathrm{HR}=$ heart rate: $\mathrm{MAP}=$ mean arterial pressure. ${ }^{*} \mathrm{P}<0.01$ vs SP (one-way ANOVA).
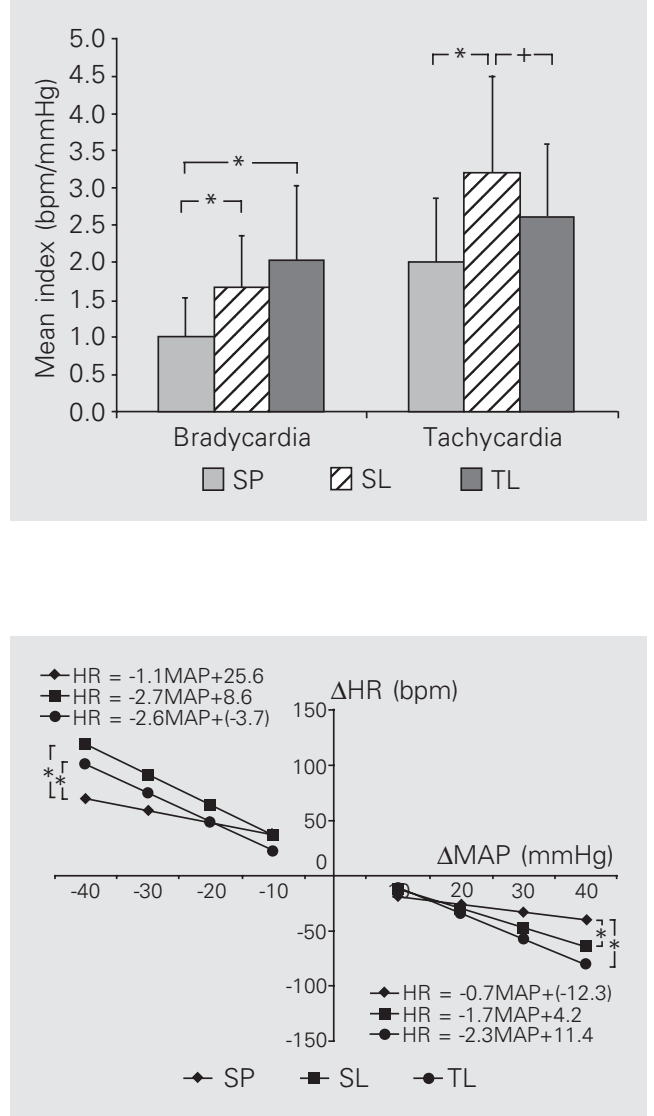

We and others have demonstrated that ET attenuates the development of hypertension in SHR $(9,10,15,18)$. Presently, we set specific goals for investigating the effect of combined treatment of losartan and ET in reducing high blood pressure in SHR versus losartan treatment alone. Confirming previous findings (11), losartan treatment at the dose of $10 \mathrm{mg} \mathrm{kg}^{-1}$ day $^{-1}$ provoked an important reduction in blood pressure in SHR. In addition, the present study clearly demonstrated that the reduction in blood pressure was mediated by total peripheral vascular resistance. These findings highlight the significant modulation of vascular tonus by angiotensin II, through the $\mathrm{AT}_{1}$ receptor, and hence, its role in the maintenance of hypertension in SHR.

The lack of a losartan effect on heart rate is not surprising, since Moreau et al. (31) have reported that losartan exhibits sympathoinhibitory effects at the vascular level, but not at the cardiac level. These investigators found that the tachycardiac response to spinal cord stimulation remained unaffected after treatment with losartan in SHR. Other investigators have reported that losartan resets the baroreflex control of heart rate to a lower pressure level, blocking the action of angiotensin II on $\mathrm{AT}_{1}$ receptors (32). Moreover, the present study confirms that losartan causes no change in cardiac output or stroke volume as previously demonstrated $(11,31)$.

ET showed no additive effect in reducing blood pressure of SHR when combined with losartan treatment. Even though an associative effect of losartan and ET has been shown to improve renal function and insulin sensitivity in rats with renal failure and type 2 diabetes mellitus, respectively, some hypotheses for the lack of a synergistic effect of ET on blood pressure could be raised. Firstly, losartan might have reduced blood pressure to the lowest levels, which minimized the antihypertensive effects of ET in SHR. Thus, it is possible that the combination of ET with 
lower doses of losartan will provide an additive effect on blood pressure in SHR. In fact, some investigators (16) reported a synergistic effect on resting caudal blood pressure in young male SHR when moderate ET was associated with small doses of some classes of antihypertensive drugs (reserpine, chlorothiazide and hydralizine). Nevertheless, the hypothesis that ET in addition to low doses of $\mathrm{AT}_{1}$ receptor blockers has a synergistic effect on blood pressure and associated autonomic controls remains to be tested. Secondly, the effect of ET on established severe hypertension is limited. Even though previous studies including those from our group $(15,16,18,33)$ demonstrated that ET plays a role by preventing the development of hypertension in young SHR (2-4 weeks of age), only very few studies were able to detect a reduction of arterial pressure after ET in adult SHR (8-12 weeks of age) with established severe hypertension $(34,35)$.

Both ET $(18,19)$ and losartan $(36)$ can separately improve baroreflex sensitivity in SHR. In the present investigation, we observed that losartan improved the baroreflex sensitivity for bradycardia and tachycardia in SHR. However, ET showed no additive effect in improving baroreflex sensitivity when combined with losartan.

It is well understood that blood pressure levels are under baroreflex control on a beatto-beat basis. Therefore, it is reasonable to speculate that the improved baroreflex sensitivity for heart rate control observed here in the SL and TL groups could be extended to a decrease in peripheral vascular resistance, and consequently, to a reduction in blood pressure levels of SHR. However, the present study provides no evidence of a causeeffect relationship between improved baroreflex sensitivity and these physiological changes.

The mechanisms underlying the improvement of arterial baroreflex sensitivity after treatment with losartan alone or in combination with ET could not be directly elucidated in the present study. However, we may suggest some potential mechanisms. First, we must emphasize that decreased blood pressure levels can restore, in part, the baroreflex sensitivity for bradycardia, since Moreira et al. (37) reported a restoration of baroreflex bradycardia after reversal of hypertension in renal hypertensive rats. Secondly, we can suggest that losartan attenuates the central effects of angiotensin II and consequently improves baroreflex sensitivity, as previously demonstrated in SHR and coarcted hypertensive rats $(17,36)$. Finally, since angiotensin II acts on specific receptors within the ventrolateral medulla, provoking a tonic excitatory action (38) and further modulation of the nucleus of the solitary tract (39), it is possible that $\mathrm{AT}_{1}$ receptor blockade largely explains the improvement in baroreflex sensitivity in SHR. In fact, losartan seems to normalize the baroreflex sensitivity for heart rate by the blockade of type 1 receptors of angiotensin II in central areas accessible orally or intracerebroventricularly (36).

In the present study, losartan treatment provoked a reduction of 12 and 9\% in left ventricular weight to body weight ratio in the SL and TL groups, respectively. Similar data were obtained by Mizuno et al. (12) who reported that losartan provoked an $11 \%$ reduction in left ventricular weight in SHR. The regression of cardiac hypertrophy observed in the present investigation could be explained by the antifibrotic, antihypertrophic and antiapoptotic effect of angiotensin II receptor blockers $(13,14,23)$. These results are of clinical relevance because they indicate that $\mathrm{AT}_{1}$ receptor blockade tends to reduce left ventricular weight towards normal levels in essential hypertension.

$\mathrm{AT}_{1}$ receptor blockade with losartan improves arterial baroreflex sensitivity for heart rate in SHR, concomitant with a reduction of blood pressure and a decrease in left ventricular weight in this model of genetic hyper- 
tension. In addition, the present study demonstrates that ET has no synergistic effect on blood pressure in established severe hypertension when combined with a high dose of losartan.

\section{Acknowledgments}

The authors thank Merck \& Co., Inc., Rahway, NJ, USA, and Fundação E.J. Zerbini, São Paulo, for supporting this study.

\section{References}

1. Flack JM, Casciano R, Casciano J, Doyle J, Arikian S, Tang S \& Arocho R (2002). Cardiovascular disease costs associated with uncontrolled hypertension. Management Care Interface, 15: 28-36.

2. Flack JM, Ferdinand KC \& Nasser AS (2003). Epidemiology of hypertension and cardiovascular disease in African Americans. Journal of Clinical Hypertension, 5: 5-11.

3. Moore MA (2002). Improving the managed care of hypertension with angiotensin II antagonists. American Journal of the Medical Sciences, 323: 25-33.

4. Israili ZH (2000). Clinical pharmacokinetics of angiotensin II (AT1) receptor blockers in hypertension. Journal of Human Hypertension, 14: S73-S86.

5. Engstrom G, Hedblad B \& Janzon L (1999). Hypertensive men who exercise regularly have lower rate of cardiovascular mortality. Journal of Hypertension, 17: 737-742.

6. Collins HL, Rodenbaugh DW \& DiCarlo SE (2000). Daily exercise attenuates the development of arterial blood pressure related cardiovascular risk factors in hypertensive rats. Clinical and Experimental Hypertension, 22: 193-202.

7. Doggrell AS (2003). Losartan for LIFE in hypertension with left ventricular hypertrophy? Expert Opinion on Pharmacotherapy, 4: 115-118.

8. Dahlof B, Devereux RB, Kjeldsen SE et al. (2002). Cardiovascular morbidity and mortality in the Losartan Intervention For Endpoint reduction in hypertension study (LIFE): a randomised trial against atenolol. Lancet, 359: 995-1003.

9. Rizzoni D, Porteri E, Piccoli A, Castellano M, Bettoni G, Muiesan ML, Pasini G, Guelfi D, Mulvany MJ \& Rosei EA (1998). Effects of losartan and enalapril on small artery structure in hypertensive rats. Hypertension, 32: 305-310.

10. Morton JJ, Beattie EC \& MacPherson F (1992). Angiotensin II receptor antagonist losartan has persistent effects on blood pressure in the young spontaneously hypertensive rat: lack of relation to vascular structure. Journal of Vascular Research, 29: 264-269.

11. Cody RJ, Binkley PF, Haas GJ \& Brown DM (1995). Acute myocardial and vascular responses to specific angiotensin II antagonism in the spontaneously hypertensive rat. American Journal of Hypertension, 8: 500-508.

12. Mizuno K, Tani M, Hashimoto S, Niimura S, Sanada H, Watanabe H, Ohtsuki M \& Fukuchi S (1992). Effects of losartan, a nonpeptide angiotensin II receptor antagonist, on cardiac hypertrophy and the tissue angiotensin II content in spontaneously hypertensive rats. Life Sciences, 51: 367-374.

13. Yu G, Liang X, Xie X, Su M \& Zhao S (2001). Diverse effects of chronic treatment with losartan, fosinopril, and amlodipine on apoptosis, angiotensin II in the left ventricle of hypertensive rats. International Journal of Cardiology, 81: 123-129.

14. Omvik P, Gerdts E, Myking OL \& Lund-Johansen P (2000). Longterm central hemodynamic effects at rest and during exercise of losartan in essential hypertension. American Heart Journal, 140:
624-630.

15. Véras-Silva AS, Mattos KC, Gava NS, Brum PC, Negrão CE \& Krieger EM (1997). Low-intensity exercise training decreases cardiac output and hypertension in spontaneously hypertensive rats. American Journal of Physiology, 273: H2627-H2631.

16. Tipton $C M$, Matthes RD, Marcus KD, Rowlett KA \& Leininger JR (1983). Influences of exercise intensity, age, and medication on resting systolic blood pressure of SHR populations. Journal of Applied Physiology, 55: 1305-1310.

17. Kawano Y, Yoshida K, Matsuoka H \& Omae $T$ (1994). Chronic effects of central and systemic administration of losartan on blood pressure and baroreceptor reflex in spontaneously hypertensive rats. American Journal of Hypertension, 7: 536-542.

18. Da Silva GJJ, Brum PC, Negrão CE \& Krieger EM (1997). Acute and chronic effects of exercise on baroreflexes in spontaneously hypertensive rats. Hypertension, 30: 714-719.

19. Brum PC, Da Silva GJ, Moreira ED, Ida F, Negrão CE \& Krieger EM (2000). Exercise training increases baroreceptor gain sensitivity in normal and hypertensive rats. Hypertension, 36: 1018-1022.

20. Kokkinos PF, Narayan P \& Papademetriou V (2001). Exercise as hypertension therapy. Cardiology Clinics, 19: 507-516.

21. Ehsani AA (2001). Exercise in patients with hypertension. American Journal of Geriatric Cardiology, 10: 253-259.

22. Hagberg JM, Park JJ \& Brown MD (2000). The role of exercise training in the treatment of hypertension: an update. Sports Medicine, 30: 193-206.

23. Oddie CJ, Dilley RJ, Kanellakis P \& Bobik A (1993). Chronic angiotensin II type 1 receptor antagonism in genetic hypertension: effects on vascular structure and reactivity. Journal of Hypertension, 11: 717-724.

24. Radaelli A, Piepoli M, Adamopoulos S, Pipilis A, Clark SJ, Casadei B, Meyer TE \& Coats AJS (1992). Effects of mild physical activity, atenolol and the combination on ambulatory blood pressure in hypertensive subjects. Journal of Hypertension, 10: 1279-1282.

25. Kelemen MH, Effron MB, Valenti AS \& Stewart KJ (1990). Exercise training combined with antihypertensive drug therapy. Effects on lipids, blood pressure, and left ventricular mass. Journal of the American Medical Association, 263: 2766-2771.

26. Tipton CM, Matthes RD, Callahan A, Tcheng TK \& Lais LT (1977). The role of chronic exercise on resting blood pressure of normotensive and hypertensive rats. Medicine and Science in Sports, 9: 168177.

27. Kohzuki M, Kamimoto M, Wu Xue-Min et al. (2001). Renal protective effects of chronic exercise and antihypertensive therapy in hypertensive rats with chronic renal failure. Journal of Hypertension, 19: 1877-1882.

28. Ishizawa K, Yoshizumi M, Tsuchiya K, Takishita E, Nakaya Y, Kishi K, Ebina $Y$, Houchi $H$, Minakuchi $K$ \& Tamaki $T$ (2001). Effects of losartan in combination with or without exercise on insulin resistance in Otsuka Long-Evans Tokushima fatty rats. European Journal 
of Pharmacology, 430: 359-367.

29. Scholander PF (1947). Analyzer for accurate estimation of respiratory gases in one-half cubic centimeter samples. Journal of Biological Chemistry, 167: 235-250.

30. Smyth HS, Sleight P \& Pickering GW (1969). Reflex regulation of arterial pressure during sleep in man. A quantitative method of assessing baroreflex sensitivity. Circulation Research, 24: 109-121.

31. Moreau N, Richer C, Vincent MP \& Giudicelli JF (1993). Sympathoinhibitory effects of losartan in spontaneously hypertensive rats. Journal of Cardiovascular Pharmacology, 22: 126-134.

32. Kumagai K \& Reid IA (1994). Angiotensin II exerts differential actions on renal nerve activity and heart rate. Hypertension, 24: 451456.

33. Tipton CM, Sebastian LA, Overton JM, Woodman CR \& Williams SB (1991). Chronic exercise and its hemodynamic influences on resting blood pressure of hypertensive rats. Journal of Applied Physiology, 71: 2206-2210.

34. Amaral SL, Zorn TMT \& Michelini LC (2000). Exercise training normalizes wall-to-lumen ratio of the gracilis muscle arterioles and reduces pressure in spontaneously hypertensive rats. Journal of Hypertension, 18: 1563-1572.

35. Amaral SL, Silveira NP, Zorn TMT \& Michelini LC (2001). Exercise training causes skeletal muscle venular growth and alters hemodynamic responses in spontaneously hypertensive rats. Journal of Hypertension, 19: 931-940.

36. Santos CM, Pontieri V, Leomil Neto M \& Michelini LC (1995). Losartan improves baroreflex control of heart rate of coarcted hypertensive rats. American Journal of Physiology, 269: H812-H818.

37. Moreira ED, Ida F \& Krieger EM (1990). Reversibility of baroreceptor hyposensitivity during reversal of hypertension. Hypertension, 15: 791-796.

38. Sasaki S \& Dampney RA (1990). Tonic cardiovascular effects of angiotensin II in the ventrolateral medulla. Hypertension, 15: 274283.

39. Andresen MC \& Kunze DL (1994). Nucleus tractus solitarius - gateway to neural circulatory control. Annual Review of Physiology, 56 : 93-116. 